\title{
Testing the comparability and interpretability of the revised professional practice environment scale - Filipino version
}

\author{
Al D. Biag* \\ School of Nursing and Allied Medical Sciences, Holy Angel University, Philippines
}

Received: November 1, 2021

Accepted: January 10, 2022

Online Published: February 21, 2022

DOI: $10.5430 /$ jnep.v12n6p31

URL: https://doi.org/10.5430/jnep.v12n6p31

\begin{abstract}
Background and aims: The Revised Professional Practice Environment (RPPE) Scale is a 39-item four Likert scale-rated questionnaire. The US-based Massachusetts General Hospital developed it as a measure of nurses' leadership and autonomy over practice, relationship with physicians, control over practice, communication about patients, teamwork, handling of disagreement and conflict, internal work motivation, and cultural sensitivity. The RPPE Scale has been translated into several languages but Filipino. The aim of this paper was to translate the RPPE Scale to the Filipino language in order to establish an initial evidence for construct equivalence between it and the original version.

Methods: Methodological design was used in the study following a four-step translation process. The data collection commenced in 2020 .

Results: The RPPE scale was subjected to forward translation in Filipino language. It was then back translated into English after which the conceptual equivalence was determined for similarity of translation and comparability of interpretation. The results based on weighted means were highly similar and highly comparable.

Conclusions: The RPPE-Filipino version demonstrated an acceptable evidence of language- and culture-specificity that is sufficiently robust for use in Philippine setting. The existence of an instrument that is comparable and similar to the original RPPE Scale paves the way for initiating nursing staff development programs that are based on the tenets of professional practice environment.
\end{abstract}

Key Words: Scale, RPPE, Positive practice environment, Filipino translation, Nurses

\section{INTRODUCTION}

The professional practice environment (PPE) is a significant factor in the quality of patient care and satisfaction of nurses. ${ }^{[1]}$ That is so, because professional practice environment supports nurses' professional judgment and competence while providing patient-centered care. ${ }^{[2]}$ Furthermore, improved professional practice environment has been documented in the empirical literature to decrease burnout and turnover among nurses. ${ }^{[3]}$

The Patient Care Services of Massachusetts General Hospital (MGH) developed a professional practice model that specifies nurses' work in different settings and care levels within the hospital. ${ }^{[4]}$ This model includes eight component elements: professional staff leadership and autonomy in practice; control over practice; staff relationships with physicians; communication about patients; teamwork; use of

\footnotetext{
*Correspondence: Al D. Biag; Email: abiag@hau.edu.ph; Address: School of Nursing and Allied Medical Sciences, Holy Angel University, Philippines.
}

Published by Sciedu Press 
problem-solving approach; enhanced internal work motivation; and delivering culturally sensitive and competent care to patients. ${ }^{[5]}$

These eight components became the conceptual basis for the development of the Professional Practice Environment (PPE) Scale. This scale was developed to include more recent dimensions of professional practice environment such as autonomy and control over practice, interpersonal communication, and culturally competent care. ${ }^{[4]}$ These dimensions were not included in earlier measures such as the Nursing Work Index (NWI), its revision (NWI-R), and Practice Environment Scale (PES). ${ }^{[6,7]}$ All these instruments were attempts to operationally measure the professional practice environment. The more recent Revised Professional Practice Environment (RPPE) scale was an outcome of further development and validation of the PPE Scale in 2005. The revision included writing of additional items for handling disagreement and conflict subscale. ${ }^{[8]}$

From then on, the RPPE scale was used in cross-cultural research. It was translated into several languages such as Finnish $;{ }^{[9-11]}$ Greek, ${ }^{[12]}$ Chinese, ${ }^{[2]}$ and Czech. ${ }^{[13]}$ The purpose in translating it from source language to these target languages is to achieve conceptual equivalence. The assumption associated with this translation is that the instrument in the target language measures the same construct as that of the original instrument. ${ }^{[14]}$

While the seminal and subsequent empirical evidence on RPPE scale showed acceptable levels of validity and reliability, these psychometric properties can never be assumed to be present in the translated versions. The translation process, then, becomes the initial effort to accruing validity evidence for construct equivalence. ${ }^{[14]}$ Before a robust factor analysis of items can be conducted to achieve construct validity of the instrument in the target language, the translation process of fers a way to minimize threats to construct equivalence. ${ }^{[15-17]}$

\section{METHOD}

\subsection{Study's aims}

The aim was to translate the Revised Professional Practice Environment (RPPE) Scale to the Filipino language in order to establish initial evidence for construct equivalence between it and the original version.

\subsection{Research design}

The research was a psychometric study; hence, it used a methodological design. The study followed the four-step translation process: forward translation, reconciliation, backward translation, and validation of the translation.

\subsection{Instrument}

The RPPE Scale is a self-administered questionnaire rated on a four-point Likert scale. It has a total of 39 items that are distributed over its eight subscales: handling disagreement and conflict; leadership and autonomy in clinical practice; internal work motivation; control over practice; teamwork; communication about patients; cultural sensitivity; and staff relationships with physicians. It can be used to elicit information about specific aspects of hospital's professional practice environment and the degree of nurses' concurrence with the scale items.

The psychometric properties (internal consistency reliability and construct validity) are almost identical in calibration and validation samples. ${ }^{[8]}$ The total score Cronbach's alphas were $r=0.93$ and $r=0.92$ for calibration and validation samples, respectively. The $59.2 \%$ variance for calibration sample and $59.7 \%$ variance for validation sample resulting from principal component analysis demonstrated evidence of construct validity. The MGH Yvonne L. Munn Center for Nursing Research granted the researcher the permission to have the RPPE Scale translated to Filipino with the condition it was not modified, and the authors are given credit in reports and publications.

\subsection{Ethical consideration}

The study was granted an exempt review by a universitybased institutional review board. The decision was contained in the notification of protocol review with assigned protocol number.

\subsection{Data collection procedure}

Data collection happened at each of the steps in the translation process. The researcher facilitated the collation of outputs from all those involved in the entire process.

\subsection{Results}

The RPPE Scale was subjected to forward translation in Filipino language. Two teachers whose specialization is Filipino language teaching and at the same time having a good command of the English language did the forward translation. They are also native speakers of the Tagalog language of which the Filipino language was lexically and grammatically based on. This premise presupposes that they are more aware of nuances involved in expressing an idea in the target language. These two translators worked independently in translating the scale, hence resulting to two Filipino versions. They translated most of the items literally and retained some English words or phrases for adoption in the Filipino version. They produced independent translations of the RPPE Scale.

The reconciliation process followed and was facilitated by 
the researcher. The role of the researcher in this stage was to act as the expert on the contents measured on the instrument. While the two translators settled on the issues on semantics and the subtleties of the target culture, the researcher provided inputs so as not to make the instrument too academic but practical for registered nurses who may better understand the items in English. The three-person committee in the reconciliation process synthesized the two versions into one. This process led to the synthesis and determination of differences as the committee agreed to consider conceptual equivalence, use of colloquial language, and clearness of expression.

The Filipino version was then translated in English by an English language professor who had not seen the RPPE Scale. This step ensured quality control in developing instruments because the back translator provides a translation that is independent from the source language version. After the back-translation, the three translators involved in the previous steps met and compared each of the items of the two English versions (i.e. source version of RPPE and its back translation). They conducted this to determine any error in translation which may result to differences in the meaning the target participants would infer. This is in accordance with the practice in cross-cultural translation of instruments that emphasized that a valid translation reflects equivalence of meaning with the original instrument.

The RPPE Scale and its back-translation were compared as to the similarity in language and their comparability of interpretation. These two dimensions provided information about the literal translation and cultural adaptation. A 7-point Likert scale was used to describe if the items are extremely comparable/extremely similar (rated as 1) or not at all comparable/not at all similar (7). This step resulted to the final version of the scale in Filipino language (RPPE Scale-Filipino version). The three translators involved in the previous steps and seven nurses working in hospitals participated in this step. Their responses were analyzed by determining the weighted mean in each of the items. They specifically rated the instrument's literal translation and comparability of interpretation or cultural adaptation. As for literal translation, the mean response $(M=1.70)$ is interpreted as highly similar. In terms of cultural adaptation, the aggregate interpretation of responses $(\mathrm{M}=1.45)$ was interpreted as highly comparable (see the Appendix). This means that the back translation version has essentially achieved the conceptual and item equivalence with the original scale.

\section{Discussion}

Translation is the most feasible approach to using instruments in other countries and cultures. Cross-cultural research re- quires thoughtful consideration of the use of language and other cultural factors to demonstrate comparable validity and reliability of the translated instrument. ${ }^{[18]}$ There are, however, downsides to translation that weaken validity and some of them are not easily identifiable unless a stringent methodological design is implemented. ${ }^{[14]}$ Thus, failure to follow the standards may result in study findings with serious validity issues.

When translating instruments to other languages, it is imperative to address issues related to language and cultural adaptation. The challenge, though, is on retaining the sense and intent of the original instrument in its translation to target language. Apart from this, cultural relevance and clarity must be evident on the translated instrument. This, therefore, underscores the objective of achieving not much of a literal but a cultural translation of the instrument in the target language.

The process of translation adds to the building of construct validity on the instrument in the target language. Through the inputs of language experts and possible target participants, the instrument's construct equivalence is established. ${ }^{[19]}$ The possibility of non-invariance of instrument items in the target language is lessened in this process. ${ }^{[20]}$ This is further established with the post-translation statistical analysis of the instrument through the data collected from the research sample. ${ }^{[21]}$

\section{Conclusion}

The translation procedures used to produce the RPPEFilipino version addressed the possibility of translation errors. Noteworthy, though, that the equivalence of meaning between the RPPE Scale and the RPPE Scale-Filipino does not ensure equivalence in the performance of the target population (nurses). This means that while a certain level of confidence can be inferred from a validated instrument, it cannot be assumed that the normative data used to build the original instrument are pertinent to the population for whom the translation is intended to. It is therefore recommended that translated instrument be subjected to psychometric testing for its validity and reliability.

\section{Implications for nursing management}

The establishment of an instrument that is comparable and similar to the original version like the case of the RPPE Scale - Filipino version provides an impetus for nursing administrators to consider using the concepts of professional practice environment as a basis for several activities in leading and managing nursing staff. The RPPE Scale can be used as a performance evaluation tool for nurses and as such can be a basis for initiating a staff development program for them. 
Its dimensions such as handling disagreement and conflict, leadership and autonomy in clinical practice, internal work motivation, control over practice, teamwork, communication about patients, cultural sensitivity, and staff relationships with physicians could provide nurse managers a rich infor- mation about their staff's dispositions, performance, and opportunities for improvement.

\section{CONFlicts of InTERest Disclosure}

The author declares that there is no conflict of interest.

\section{REFERENCES}

[1] Meier A, Ives Erickson J, Snow N, et al. Nurse and patient satisfaction. Journal of Nursing Administration. 2019; 49(11): 520-522. PMid:31651609 https://doi.org/10.1097/NNA.0000000000 000814

[2] Guarino AJ, Kelly L, Ives Erickson J, et al. The psychometric properties of the Chinese version of the Revised Professional Practice Environment Scale (RPPE): Utilized in assessing quality control. Journal of Community Medicine and Public Health Care. 2016; 3(19): 1-4. https ://doi.org/10.24966/CMPH-1978/100019

[3] Aiken LH, Sloane DM, Bruyneel L, et al. Nurses' reports of working conditions and hospital quality of care in 12 countries in Europe. International Journal of Nursing Studies. 2013; 50: 143-153. PMid:23254247 https://doi.org/10.1016/j.ijnurstu. 201 2.11.009

[4] Ives Erickson J, Duffy ME, Gibbons MP, et al. Development and psychometric evaluation of the Professional Practice Environment (PPE) Scale. Journal of Nursing Scholarship. 2004; 36(3): 279-285. PMid:15495499 https : //doi.org/10.1111/j.1547-5069. 20 $04.04050 . \mathrm{x}$

[5] Ives Erickson J, Ditomassi M. Professional practice model: Strategies for translating models into practice. Nursing Clinics of North America. 2011; 46(1): 35-44. PMid:21320659 https : //doi .org/ 10.1016/j.cnur. 2010.10.011

[6] Aiken LH, Patrician PA. Measuring organizational traits of hospitals: The Revised Nursing Work Index. Nursing Research. 2000; 49(3): 146-153. PMid:10882319 https://doi.org/10.1097/00 006199-200005000-00006

[7] Lake ET. Development of the Practice Environment Scale of the Nursing Work Index. Research in Nursing and Health. 2002; 25(3): 176188. PMid:12015780 https ://doi .org/10.1002/nur.10032

[8] Ives Erickson J, Duffy ME, Ditomassi M, et al. Psychometric Evaluation of the Revised Professional Practice Environment (RPPE) Scale. Journal of Nursing Administration. 2009; 39(5): 236-243. PMid:19423989 https ://doi.org/10.1097/NNA.0b013e3181 a23d14

[9] Charalambous A, Katajisto J, Valimaki M, et al. Individualised care and the professional practice environment: Nurses' perceptions. International Nursing Review. 2010; 57(4): 500-507. PMid:21050203 https://doi.org/10.1111/j.1466-7657.2010.00831.x

[10] Suhonen R, Efstathiou G, Tsangari H, et al. Patients' and nurses' perceptions of individualised care: An international comparative study. Journal of Clinical Nursing. 2012; 21(7-8): 1155-67. PMid:21884558 https://doi.org/10.1111/j.1365-2702.2011.03833.x

[11] Papastavrou E, Efstathiou G, Acaroglu R, et al. A seven country comparison of nurses' perceptions of their professional practice environment. Journal of Nursing Management. 2012; 20(2): 236-248.
PMid:22050114 https : //doi.org/10.1111/j.1365-2834. 20 11.01289. $\mathrm{x}$

[12] Papastavrou E, Andreou P, Middleton N, et al. Translation and validation of the Revised Professional Practice Environment Questionnaire in the Greek language. Journal of Nursing Measurement. 2015; 23(3): 112-27. https://doi.org/10.1891/1061-3749.23.3.112

[13] Zeleníková R, Jarošová D, Plevová I, et al. Nurses' perceptions of professional practice environment and its relation to missed nursing care and nurse satisfaction. International Journal of Environmental Research and Public Health. 2020; 17(11): 3805. PMid:32471133 https://doi.org/10.3390/ijerph17113805

[14] Hawkins M, Cheng C, Elsworth GR, et al. Translation method is validity evidence for construct equivalence: Analysis of secondary data routinely collected during translations of the Health Literacy Questionnaire (HLQ). BMC Medical Research Methodology. 2020; 20(130). PMid:32456680 https://doi.org/10.1186/s1 2874-020-00962-8

[15] American Educational Research Association, American Psychological Association, \& National Council on Measurement in Education (Eds.). (2014). Standards for educational and psychological testing. American Educational Research Association.

[16] Acquadro C, Patrick DL, Eremenco S, et al. Emerging good practices for Translatability Assessment (TA) of Patient-reported outcome (PRO) measures. Journal of Patient-reported Outcomes. 2018; 2(8). PMid:29757337 https : //doi.org/10.1186/s41687-018-003 5-8

[17] Hawkins M, Elsworth GR, Osborne RH. Application of validity theory and methodology to patient-reported outcome measures (PROMs): Building an argument for validity. Quality of Life Research,. 2018; 27(7): 1695-1710. PMid:29464456 https://doi. org/10.1007/s11136-018-1815-6

[18] Ohrbach R, Bjomer J, Jezewski MA, et al. Guidelines for establishing cultural equivalency of instruments. State University of New York at Buffalo. 2013. Available from: https://ubwp.buffalo.edu/r dc-tmdinternational/wp-content/uploads/sites/58/20 17/01/Guidelines-for-Translation-and-Cultural-Equ ivalency-of-Instruments-2013_05_118608.pdf

[19] Epstein J, Santo RM, Guillemin F. A review of guidelines for crosscultural adaptation of questionnaires could not bring out a consensus. Journal of Clinical Epidemiology. 2015; 68(4): 435-41. PMid:25698408 https://doi.org/10.1016/j.jclinepi. 201 4.11.021

[20] Millsap RE. Statistical approaches to measurement invariance. London: Routledge. 2012.

[21] Kane MT. Validating the interpretations and uses of test scores. Journal of Educational Measurement. 2013; 50(1): 1-73. https: //doi.org/10.1111/jedm. 12000 